ABSTRACT: Neuromuscular electrical stimulation (NMES) involves the use of electrical current to facilitate contraction of skeletal muscle. However, little is known concerning the effects of varying stimulation parameters on muscle function in humans. The purpose of this study was to determine the extent to which varying pulse duration and frequency altered torque production and fatigability of human skeletal muscle in vivo. Ten subjects underwent NMES-elicited contractions of varying pulse frequencies and durations as well as fatigue tests using stimulation trains of equal total charge, yet differing parametric settings at a constant voltage. Total charge was a strong predictor of torque production, and pulse trains with equal total charge elicited identical torque output. Despite similar torque output, higherfrequency trains caused greater fatigue. These data demonstrate the ability to predictably control torque output by simultaneously controlling pulse frequency and duration and suggest the need to minimize stimulation frequency to control fatigue.

Muscle Nerve 35: 504-509, 2007

\title{
IMPACT OF VARYING PULSE FREQUENCY AND DURATION ON MUSCLE TORQUE PRODUCTION AND FATIGUE
}

\author{
CHRIS M. GREGORY, PhD, ${ }^{1}$ WARREN DIXON, PhD, ${ }^{2}$ and C. SCOTT BICKEL, PhD ${ }^{3}$ \\ ${ }^{1}$ Brain Rehabilitation Research Center, North Florida / South Georgia Veterans Health System, and \\ Department of Physical Therapy, University of Florida, Gainesville, Florida 32610, USA \\ ${ }^{2}$ Department of Mechanical and Aerospace Engineering, University of Florida, \\ Gainesville, Florida, USA \\ ${ }^{3}$ Department of Physical Therapy, University of Alabama at Birmingham, \\ Birmingham, Alabama, USA \\ Accepted 24 October 2006
}

Neuromuscular electrical stimulation (NMES) involves the use of electrical current to facilitate contraction of skeletal muscle. It is commonly used during rehabilitation to accomplish a variety of goals and often prescribed to treat muscle atrophy or impaired motor control associated with orthopedic and neurological damage or joint motion dysfunction. ${ }^{2}$ In addition, NMES can serve as a means of compensating for loss of voluntary control of skeletal muscles as well as facilitating exercise to restore or supplement function lost due to disease or injury. $3,15,16,18$ Nevertheless, inherent disadvantages surround the application of NMES, including decreased subject tolerance and, most notably, rapid onset of fatigue relative to voluntary contractions. ${ }^{12,24}$ It is the latter

\footnotetext{
Abbreviations: MVIC, maximum voluntary isometric contraction; NMES, neuromuscular electrical stimulation; PPI, present pain intensity

Key words: electrical stimulation; fatigue; muscle; pain

Correspondence to: C. M. Gregory; e-mail: cgregory@phhp.ufl.edu

Published 2007 by Wiley Periodicals, Inc. This article is a US Government work and, as such, is in the public domain in the United States of America. Published online 17 January 2007 in Wiley InterScience (www.interscience. wiley.com). DOI 10.1002/mus.20710
}

of these that results in the greatest limitations when NMES applications are used to elicit functional activities.

The characteristics of electrical stimulation known to impact external torque production include the intensity (i.e., amplitude or voltage), frequency, and duration of pulses. ${ }^{2}$ The most widely studied of these characteristics are frequency and intensity. Increasing the intensity results in recruitment of additional motor units, thereby increasing torque output. ${ }^{1}$ Increasing stimulation frequency will also increase torque output. This phenomenon holds true to the point that a tetanic contraction is achieved (i.e., any further increase in frequency will not increase torque). Subtetanic increases in stimulation frequency increase torque output by maximizing the amount of torque each individual motor unit can produce without affecting recruitment. ${ }^{4}$

Previous studies suggest that both high stimulation frequencies and intensities may accelerate the rate and level of fatigue during NMES. ${ }^{19,22}$ As such, alternative methods of attaining or maintaining torque output at desired levels using lower activation 
frequencies and intensities, thereby potentially altering fatigability, would be beneficial in the design and implementation of optimal stimulation protocols. Previous studies have examined the impact of using reduced frequencies and increasing current intensity to attain a given torque level, and shown improvements in fatigue resistance using this approach. ${ }^{5}$ The physiological explanation for these results involves recruiting more motor units at lower firing frequencies to achieve a given torque output. As such, these studies emphasize the importance of stimulation frequency over intensity in causing fatigue. However, to our knowledge, no data exist on the impact of varying pulse duration, either alone or in combination with other parameters, on torque production and fatigability during NMES, even though an understanding of the interaction of these stimulation parameters and their impact on muscle function seems valuable. Therefore, the purpose of this study was to examine the interrelationship of pulse duration and pulse frequency at constant stimulation intensity on torque production and fatigue of human skeletal muscle in vivo.

\section{MATERIALS AND METHODS}

Subjects. Ten subjects $(29.9 \pm 6.7$ years, $174.2 \pm 7.4$ $\mathrm{cm}, 72.7 \pm 11.6 \mathrm{~kg} ; 8 \mathrm{men})$ participated in this study. Criteria for participation included: (1) 18-50 years of age, (2) recreationally active, (3) no history of orthopedic or neurological injury that might affect lowerextremity muscle function, and (4) no known medical conditions that contraindicated NMES. Prior to participating in the study, written informed consent was obtained from all subjects, as approved by our Institutional Review Board.

Isokinetic Dynamometry. Torque measurements were obtained from the quadriceps muscle group using an isokinetic dynamometer (Biodex, Shirley, New York). Subjects were seated in an upright chair with hips and knees flexed to $\sim 80^{\circ}$. The axis of the dynamometer was aligned with the knee joint line and the leg was secured to the lever arm. Proximal stabilization was achieved with straps around the chest, waist, and thigh, as previously described. ${ }^{17}$ Prior to data collection, subjects were allowed to perform several warm-up contractions. Next, a value for maximum voluntary isometric contraction (MVIC) was determined. MVIC was defined as the peak isometric torque achieved during three consecutive contractions $(\sim 5$-s contractions separated by $120 \mathrm{~s}$ of rest). In the event that the peak torque values differed by more than $5 \%$, additional contractions were performed. Contraction in- tensity for subsequent NMES testing was calculated relative to each subjects' MVIC.

Electrical Stimulation. Bipolar self-adhesive neuromuscular stimulation electrodes were placed over the distal-medial and proximal-lateral portion of the quadriceps muscle group. Stimulation pulses were delivered using a Grass S8800 stimulator with a Grass Model SIU8T stimulus isolation unit (Grass Instruments, Quincy, Massachusetts) and data digitized at 200 samples per second. Intensity was set at a voltage that elicited $\sim 50 \%$ of each subjects' MVIC using a $70-\mathrm{Hz} / 600-\mu$ s pulse train of $500-\mathrm{ms}$ duration. Prior to data collection, five trains (500-ms train duration) were delivered at the aforementioned settings to potentiate the quadriceps muscle group. Next, 500 -ms pulse trains were delivered using all possible combinations of frequencies $(10,20,30,40,50,60$, 70 , and $100 \mathrm{~Hz})$ and pulse durations $(100,200,300$, $400,500,600$, and $700 \mu \mathrm{s})$ at the predetermined voltage. A minimum of $30 \mathrm{~s}$ rest was given between each contraction. Pulses were delivered in random order with the exception that the $70-\mathrm{Hz} / 600-\mu$ s train was given at the beginning, middle, and end of the protocol in an effort to assess fatigue during the testing session. A total of 58 NMES-induced contractions were elicited. In addition, fatigue tests were performed during subsequent sessions separated by 48-96 h. Fatigue tests were performed using different parametric settings of equal total charge (i.e., $50-\mathrm{Hz} / 200-\mu$ s vs. $20-\mathrm{Hz} / 500-\mu \mathrm{s})$ at the same voltage used previously and a $50 \%$ duty cycle (1-s on / 1-s off). Fatigue tests were $2 \mathrm{~min}$ in duration (i.e., 60 total contractions).

Present Pain Intensity (PPI). Following fatigue tests, subjects rated PPI using a visual analog scale as part of the short-form McGill Pain Questionnaire. ${ }^{20}$ The scale ranges from 0 to $100 \mathrm{~mm}$ with the zero value representing "no pain" and the $100 \mathrm{~mm}$ value representing the "worst possible pain."

Data Analyses. Torque values obtained for each combination of pulse duration and frequency were used to construct torque-duration and torque-frequency curves. Torque values obtained were normalized to trains elicited using the highest frequency and longest pulse duration (i.e., $70-\mathrm{Hz} / 600-\mu \mathrm{s}$ ) to construct the normalized torque-duration and torque-frequency curves, respectively. Next, regression analysis was used to determine the relationship between torque produced during parameter titration and total charge (product of pulse duration and pulse frequency; $\left.\mathrm{V} * \mathrm{~s}^{-6}\right)$. T-tests were used to determine whether differ- 
ences existed between torque values elicited by trains of similar total charge yet varying parametric settings. Finally, $t$-tests were used to determine whether differences existed in the torque produced or the discomfort reported between contractions elicited by pulse trains of different parametric settings yet equal total charge. For all tests performed, the level of significance was set at $\alpha=0.05$.

\section{RESULTS}

Normalized torque data were plotted over a range of pulse frequencies $(10-100 \mathrm{~Hz})$ at different durations $(100-600 \mu \mathrm{s})$ to construct the torquefrequency curve (Fig. 1). In addition, a torqueduration curve was constructed across this same range of pulse durations and averaged across the different frequencies (Fig. 2).

Total charge, the product of pulse frequency and pulse duration $\left(\mathrm{V} * \mathrm{~s}^{-6}\right)$, is a strong predictor of external torque production during NMES-induced contractions in human skeletal muscle. Total charge explained $\sim 98 \%$ of the variance in external torque production over a range of pulse durations $(200-600 \mu \mathrm{s})$ and pulse frequencies $\left(20-60 \mathrm{~Hz} ; \mathrm{y}=-5 \mathrm{E}-10 \mathrm{x}^{2}+3 \mathrm{E}-\right.$ $05 x+0.3262, R^{2}=0.9812$; Fig. 3 ). Of note, no differences were observed in torque production between $70-\mathrm{Hz} / 600-\mu$ s trains delivered throughout the testing protocol. Thus, fatigue of the quadriceps femoris muscle group was not a factor in the relationships found. In this study we limited the range of stimulation frequencies used to determine this relationship so as to avoid those that exist on the plateau of the torque-frequency relationship. Specifically, frequencies where an additional $10-\mathrm{Hz}$ increase did not significantly increase torque production were excluded. In addition, the lowest pulse frequency $(10 \mathrm{~Hz})$ and duration $(100 \mu \mathrm{s})$

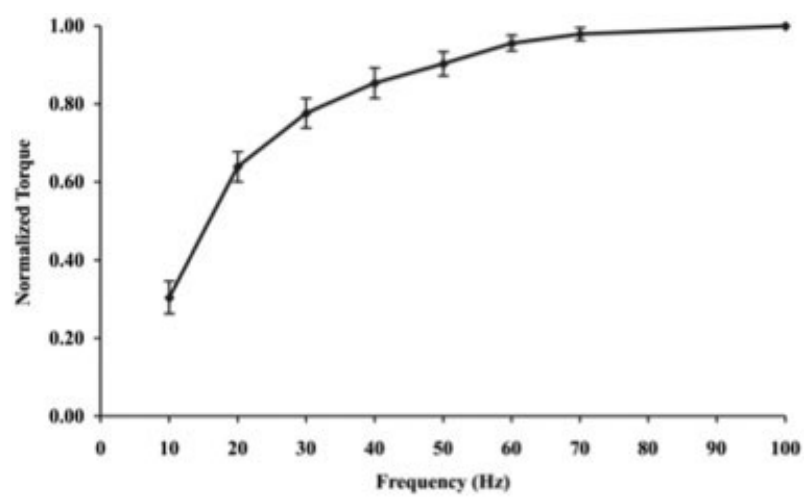

FIGURE 1. Normalized torque-frequency relationship of the quadriceps muscle group averaged over a range of pulse durations $(100-600 \mu \mathrm{s})$ at constant current amplitude. Values represent mean \pm SEM.

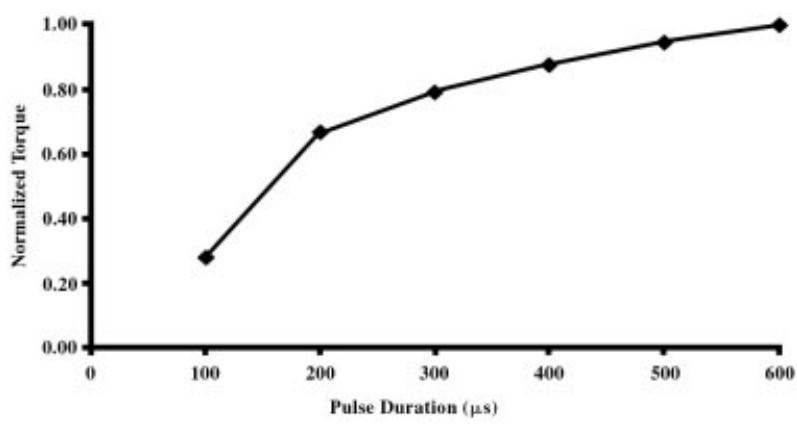

FIGURE 2. Normalized torque-duration curve averaged over a range of pulse frequencies $(10-100 \mathrm{~Hz})$ at constant current amplitude. Values represent mean \pm SEM.

were excluded from this relationship. However, as indicated in Figure 3, the relationship between total charge and external torque production plotted using only these lowest parameter values resulted in a separate but similarly strong relationship, with total charge explaining $\sim 92 \%$ of the variance in external torque production $\left(\mathrm{y}=-2 \mathrm{E}-09 \mathrm{x}^{2}+4 \mathrm{E}-05 \mathrm{x}+0.0943, \mathrm{R}^{2}=\right.$ 0.9162; Fig. 3).

No differences were found in the amount of torque produced by trains of equal total charge, regardless of stimulation intensity $(P \geq 0.26$; Tables 1,2$)$. In addition, pulse trains of $20 \mathrm{~Hz} / 500 \mu \mathrm{s}(40.3 \pm 8.1 \mathrm{ft} * \mathrm{lbs})$ and $50 \mathrm{~Hz} / 200 \mu \mathrm{s}(40.3 \pm 4.7 \mathrm{ft} * \mathrm{lbs})$ elicited similar initial torques at the onset of the fatigue tests. However, the stimulation trains utilizing the lower frequency stimulation resulted in less reduction in torque (45.0 \pm $4.6 \%$ vs. $62.0 \pm 3.6 \% ; P=0.0005)$ during fatigue tests. In fact, the lower frequency setting resulted in greater

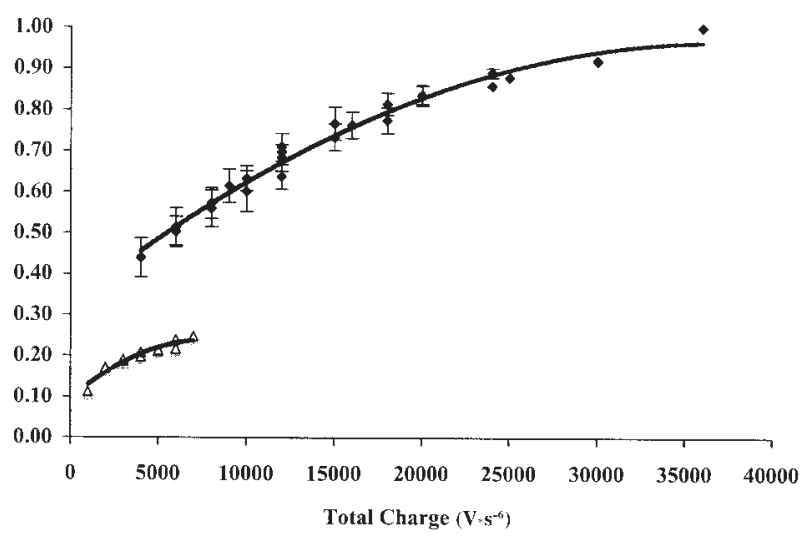

FIGURE 3. Relationship between normalized torque during a $500-\mu$ s stimulation train and total charge $\left(\mathrm{V} * \mathrm{~s}^{-6}\right)$. Filled symbols represent values over a range of frequencies $(20-60 \mathrm{~Hz})$ and pulse durations $(200-600 \mu \mathrm{s})$. Open symbols represent torque values obtained by stimulation trains of varying frequencies at a $100-\mu$ s pulse duration and over a range of pulse durations at a $10-\mathrm{Hz}$ pulse frequency. Values are mean \pm SEM. 
Table 1. Mean torque values (ft*lbs \pm SEM) obtained during 500ms stimulation trains matched by total charge elicited at all combinations of frequencies $(20-60 \mathrm{~Hz})$ and pulse durations $(200-600 \mu s)$.

\begin{tabular}{|c|c|}
\hline Total charge & Mean torque \\
\hline \multicolumn{2}{|l|}{6000} \\
\hline $20 \mathrm{~Hz} / 300 \mu \mathrm{s}$ & $41.4(5.9)$ \\
\hline $30 \mathrm{~Hz} / 200 \mu \mathrm{s}$ & $42.8(6.6)$ \\
\hline \multicolumn{2}{|l|}{8000} \\
\hline $20 \mathrm{~Hz} / 400 \mu \mathrm{s}$ & $46.1(5.6)$ \\
\hline $40 \mathrm{~Hz} / 200 \mu \mathrm{s}$ & $46.3(6.9)$ \\
\hline \multicolumn{2}{|l|}{10000} \\
\hline $20 \mathrm{~Hz} / 500 \mu \mathrm{s}$ & $50.5(5.5)$ \\
\hline $50 \mathrm{~Hz} / 200 \mu \mathrm{s}$ & $49.8(7.3)$ \\
\hline \multicolumn{2}{|l|}{12000} \\
\hline $30 \mathrm{~Hz} / 400 \mu \mathrm{s}$ & $54.6(6.3)$ \\
\hline $40 \mathrm{~Hz} / 300 \mu \mathrm{s}$ & $52.8(7.7)$ \\
\hline \multicolumn{2}{|l|}{12000} \\
\hline $20 \mathrm{~Hz} / 600 \mu \mathrm{s}$ & $57.8(7.5)$ \\
\hline $60 \mathrm{~Hz} / 200 \mu \mathrm{s}$ & $57.9(8.4)$ \\
\hline \multicolumn{2}{|l|}{15000} \\
\hline $30 \mathrm{~Hz} / 500 \mu \mathrm{s}$ & $61.9(7.3)$ \\
\hline $50 \mathrm{~Hz} / 300 \mu \mathrm{s}$ & $60.0(7.8)$ \\
\hline \multicolumn{2}{|l|}{18000} \\
\hline $30 \mathrm{~Hz} / 600 \mu \mathrm{s}$ & $65.3(7.4)$ \\
\hline $60 \mathrm{~Hz} / 300 \mu \mathrm{s}$ & $63.7(8.8)$ \\
\hline \multicolumn{2}{|l|}{20000} \\
\hline $40 \mathrm{~Hz} / 500 \mu \mathrm{s}$ & $67.6(8.1)$ \\
\hline $50 \mathrm{~Hz} / 400 \mu \mathrm{s}$ & $68.3(8.6)$ \\
\hline \multicolumn{2}{|l|}{24000} \\
\hline $40 \mathrm{~Hz} / 600 \mu \mathrm{s}$ & $71.6(8.1)$ \\
\hline $60 \mathrm{~Hz} / 400 \mu \mathrm{s}$ & $70.0(8.5)$ \\
\hline \multicolumn{2}{|l|}{30000} \\
\hline $50 \mathrm{~Hz} / 600 \mu \mathrm{s}$ & $73.8(7.9)$ \\
\hline $60 \mathrm{~Hz} / 500 \mu \mathrm{s}$ & $74.3(8.5)$ \\
\hline
\end{tabular}

Comparisons for each pair of mean torque values for a given total charge showed no differences $(P>0.05)$.

average torque values beginning with contraction 6 of 60 and continuing throughout the remainder of the test (Fig. 4).

No differences were found in the VAS pain ratings of subjects utilizing pulse trains of $20 \mathrm{~Hz} / 500 \mu \mathrm{s}$ $(15.33 \pm 2.96 \mathrm{~mm})$ or $50 \mathrm{~Hz} / 200 \mu \mathrm{s}(20.76 \pm 3.75$ $\mathrm{mm}, P=0.18)$.

\section{DISCUSSION}

The results of this study demonstrate that the product of pulse duration and pulse frequency, defined as total charge, is an extremely strong predictor of external torque production in healthy human skeletal muscle. Accordingly, pulse trains with equal total charge elicit identical torques across a range of pulse frequencies and durations. Finally, stimulation settings of equal charge that incorporate lower frequencies of activation result in less fatigue with no difference in perceived pain. Of note, a key characteristic
Table 2. Mean torque values (ft*lbs \pm SEM) obtained during 500 ms stimulation trains matched by total charge elicited across a range of pulse durations at $10 \mathrm{~Hz}$ and a range of frequencies at a pulse duration of $100 \mu \mathrm{s}$.

\begin{tabular}{cc}
\hline Total charge & Mean torque \\
\hline 2000 & \\
$10 \mathrm{~Hz} / 200 \mu \mathrm{s}$ & $14.1(3.4)$ \\
$20 \mathrm{~Hz} / 100 \mu \mathrm{s}$ & $14.9(3.6)$ \\
3000 & $15.3(3.5)$ \\
$10 \mathrm{~Hz} / 300 \mu \mathrm{s}$ & $16.8(4.4)$ \\
$30 \mathrm{~Hz} / 100 \mu \mathrm{s}$ & $16.3(3.9$ \\
4000 & $18.4(5.1)$ \\
$10 \mathrm{~Hz} / 400 \mu \mathrm{s}$ & $17.3(4.0)$ \\
$40 \mathrm{~Hz} / 100 \mu \mathrm{s}$ & $18.9(5.0)$ \\
5000 & \\
$10 \mathrm{~Hz} / 500 \mu \mathrm{s}$ & $17.6(3.8)$ \\
$50 \mathrm{~Hz} / 100 \mu \mathrm{s}$ & $21.0(5.3)$ \\
6000 & \\
$10 \mathrm{~Hz} / 600 \mu \mathrm{s}$ & \\
$60 \mathrm{~Hz} / 100 \mu \mathrm{s}$ &
\end{tabular}

Comparisons for each pair of mean torque values for a given total charge showed no differences $(\mathrm{P}>0.05)$.

of this study is that stimulation intensity was held constant at a relatively high level throughout the entire protocol in an effort to ensure substantial quadriceps muscle activation and was necessary to obtain measurable torque with the low frequency / low pulse duration trains.

The values in Tables 1 and 2 reflect the different combinations of parameters that result in identical total charge and represent some novel findings. These pairs of NMES trains, independent of magnitude, result in equal torque. For example, there was no difference in external torque between the $30-\mathrm{Hz} / 500-\mu \mathrm{s}$ and $50-\mathrm{Hz} / 300-\mu$ s trains, both representing trains with a $15,000 \mathrm{~V} * \mathrm{~s}^{-6}$ total charge. Interestingly, these two frequencies fall on the steep part of the torque-frequency curve (Fig. 1), suggesting that the torques should be

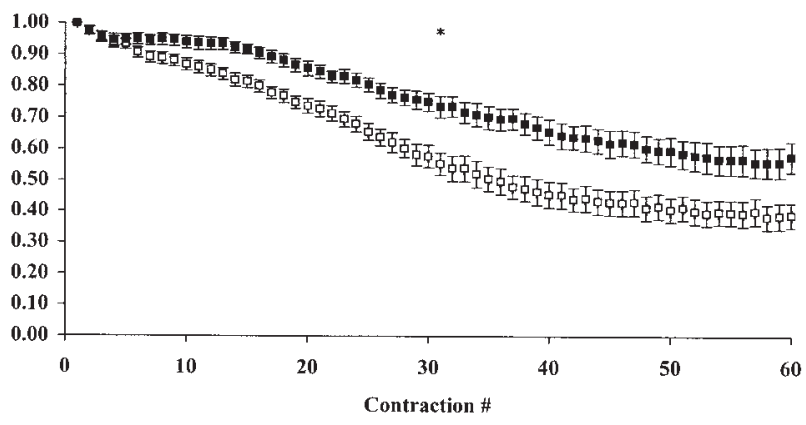

FIGURE 4. Normalized torque values for each contraction obtained during fatigue test (60 contractions; $1 \mathrm{~s}$ on: $1 \mathrm{~s}$ off) using $50 \mathrm{~Hz} / 200 \mu$ s pulses (open squares) or $20 \mathrm{~Hz} / 500 \mu$ s pulses (closed squares). *Significantly different torque values between conditions. 
quite different. However, by simply adjusting pulse duration to normalize total charge, external torque production was the same. A similar finding was seen in the $20-\mathrm{Hz} / 600-\mu$ s and $60-\mathrm{Hz} / 200-\mu$ s trains. These two frequencies are even further apart on the torque-frequency curve, yet when total charge is equalized the trains produced identical torques.

The torque-frequency relationship of the quadriceps muscle group illustrated in Figure 1 is similar to that previously reported. ${ }^{6}$ The novelty of our data lies in the fact that the curve is generated from normalizing the torque-frequency relationship over multiple pulse durations $(100-600 \mu \mathrm{s})$, owing to the inherent stability of this physiological phenomenon in nonfatigued muscle. In general, force is relatively low at the lowest frequency due to an inability of the pulses to summate action potentials and result in higher torques. However, torque increases exponentially with increases in frequency until about $60 \mathrm{~Hz}$, where torque seemingly plateaus. ${ }^{22}$ This plateau is due to calcium uptake being the rate-limiting step while muscle cross-bridge cycling is occurring at a maximal rate, resulting in the maximum torque per muscle fiber in the motor unit. ${ }^{10}$ Increasing the frequency and providing more calcium does not result in greater torque. Accordingly, energy expenditure will be high during higher frequency stimulation. Interestingly, a higher energy cost of contraction with increased frequencies, even at equal torques, has previously been reported during tetanic contractions. ${ }^{21}$ In our study, stimulation trains with higher frequencies, and presumably higher energy costs, resulted in greater muscle fatigue than lower-frequency stimulation (Fig. 4). Although an increased metabolic cost at higher frequencies seems the likely mechanism to explain the fatigue response noted, definitive data examining differences in metabolic demand of subtetanic contractions warrants future study.

In addition to a predictable response to alterations in frequency of stimulation, skeletal muscle torque production is also affected by altering pulse duration. This relationship is curvilinear in nature and is often referred to as the strength-duration curve. ${ }^{14}$ The data generated in the present study allowed us to illustrate this relationship, herein referred to as the torque-duration curve given our units of measure (Fig. 2). Similar to the torquefrequency curve, the reproducibility of the torque-duration relationship in the quadriceps muscle group is illustrated by its low variability, despite being generated from the normalized response over eight different frequencies. Although the relationship between muscle output and pulse duration is an accepted phenomenon in skeletal muscle, the mechanisms that dictate this response are understudied. We suspect that during stimulation protocols similar to those in the present study, longer pulse durations increase recruitment by more easily overcoming resistance to current flow, thereby facilitating motor unit recruitment at a given intensity, resulting in greater torque output. In addition, because firing frequency is driving calcium release/reuptake and results in widely different specific tension of muscle fibers, ${ }^{13}$ the logical assumption is that changing pulse duration results in different numbers of motor units being recruited. In fact, the potential for increasing pulse duration to result in greater muscle recruitment has recently been demonstrated. ${ }^{13}$

The intriguing part of this study is that the product of pulse duration and pulse frequency seemingly dictates external torque production (Fig. 3). However, stimulation combinations that utilized either the $10 \mathrm{~Hz}$ or $100 \mu$ s parameter had consistently low torque values. Seemingly, total charge can still predict external torque production at these levels $\left(\mathrm{R}^{2}=\right.$ 0.91 ) but the low pulse duration and low frequency severely limit the ability of the muscle to produce torque. At $10 \mathrm{~Hz}$ there is little to no summation of action potentials, so that peak torque is essentially derived from a single pulse. There may be limitations in motor unit recruitment when using $100-\mu$ s pulse durations as it has been shown that when short pulse durations are used it is necessary to increase current in order to reach threshold in peripheral nerves. ${ }^{14}$

The finding of no difference in pain intensity between the two different stimulation protocols is important. If significantly more pain was present during the stimulation patterns that elicited enhanced muscle function it would be difficult to justify incorporation of such protocols. As it is, perception of pain during NMES in healthy persons is thought to limit the application of this modality. Interestingly, the pain values reported in our subjects were on the low end of the VAS scale and were typically described as "mild" in evaluative assessments on the McGill Pain Questionnaire. Accordingly, these data do not support pain as a factor limiting the application of NMES, even at the relatively high stimulation intensities utilized in our study.

Although few data from human studies exist to explain the response of skeletal muscle to changes in pulse frequency and duration, important information to aid in the development of optimal stimulation strategies can be obtained from muscle modeling studies. Data from these studies suggest that the ability to control multiple parameters of stimulation can improve evoked responses, thus supporting the importance of the current study. Specifically, these studies have indi- 
cated that the combination of pulse-frequency modulation with pulse-width modulation can improve the transient response of skeletal muscle over modulation of either parameter alone. ${ }^{7}$ However, to our knowledge these results have never matured into a definitive relationship that could govern NMES modulation strategies in vivo. A clear need remains for practical solutions and a better understanding of the underlying mechanisms of muscle responses induced by NMES. ${ }^{22}$ We suggest that optimizing stimulation parameters during NMES-induced contractions of skeletal muscles can have a variety of applications. Limitations imposed by suboptimal parametric settings could seemingly limit the desired effects of an NMES training protocol by minimizing muscle activation or torque production as well as fatigability. Given that fatigability of muscle resulting from NMES is a major limitation to its application, the ability to account for or minimize this effect would be invaluable to NMES prescription. However, predicting or compensating for muscle fatigue is a daunting task given the complex nonlinear behaviors exhibited by the muscle response to simultaneous modulation of multiple parameters.

In conclusion, the identification of stimulation patterns that maximize muscle performance would allow more physiologically advantageous activation patterns to be used during electrical stimulation protocols. Our data suggest the importance of minimizing stimulation frequency to achieve a given absolute torque when fatigue is a concern, and demonstrate the ability to predictably control torque output by simultaneously controlling both pulse frequency and duration.

Funded in part by NIH RO1-HD048051 as well as the Department of Veterans Affairs Associate Investigator Award (573/05B1) and Center of Excellence grant (F2182C; Brain Rehabilitation Research Center) from the Rehabilitation Research and Development Service of the Department of Veterans Affairs. This work was also supported in part by a NSF Career Award to W.D. (CMS0547448). It is the result of work supported by the Office of Research and Development, Rehabilitation R\&D Service, Department of Veterans Affairs, and the Malcom Randall VA Medical Center, Gainesville, Florida.

\section{REFERENCES}

1. Adams GR, Harris RT, Woodard D, Dudley GA. Mapping of electrical muscle stimulation using MRI. J Appl Physiol 1993; 74:532-537.

2. Alon G. Principles of electrical stimulation. In: Nelson R, Hayes K, Currier D, editors. Clinical electrotherapy. Upper Saddle River, NJ: Prentice Hall; 1999.

3. Bax L, Staes F, Verhagen A. Does neuromuscular electrical stimulation strengthen the quadriceps femoris? A systematic review of randomised controlled trials. Sports Med 2005;35: 191-212.

4. Binder-Macleod SA, Halden EE, Jungles KA. Effects of stimulation intensity on the physiological responses of human motor units. Med Sci Sports Exerc 1995;27:556-565.
5. Binder-Macleod SA, Lee SCK, Baadte SA. Reduction of the fatigue induced force decline in human skeletal muscle by optimized stimulation trains. Arch Phys Med Rehabil 1997; 78:1129-1137.

6. Binder-Macleod SA, McDermond LR. Changes in the forcefrequency relationship of the human quadriceps femoris muscle following electrically and voluntarily induced fatigue. Phys Ther 1992;72:95-104.

7. Chizeck H, Lan N, Palmieri LS, Crago P. Feedback control of electrically stimulated muscle using simultaneous pulse width and stimulus period modulation. IEEE Trans Biom Eng 1991; 38:1224-1234.

8. Ding J, Lee SC, Johnston TE, Wexler AS, Scott WB, BinderMacleod SA. Mathematical model that predicts isometric muscle forces for individuals with spinal cord injuries. Muscle Nerve 2005;31:702-712.

9. Ding J, Wexler AS, Binder-Macleod SA. Mathematical models for fatigue minimization during functional electrical stimulation. J Electromyogr Kinesiol 2003;13:575-588.

10. Endoh M. Force-frequency relationship in intact mammalian ventricular myocardium: physiological and pathophysiological relevance. Eur J Pharmacol 2004;500:73-86.

11. Frey Law LA, Shields RK. Predicting human chronically paralyzed muscle force: a comparison of three mathematical models. J Appl Physiol 2006;100:1027-1036.

12. Gondin J, Guette M, Jubeau M, Ballay Y, Martin A. Central and peripheral contributions to fatigue after electrostimulation training. Med Sci Sports Exerc 2006;38:1147-1156.

13. Gorgey AS, Mahoney E, Kendall T, Dudley GA. Effects of neuromuscular electrical stimulation parameters on specific tension. Eur J Appl Physiol 2006;97:737-734.

14. Grill WM Jr, Mortimer JT. The effect of stimulus pulse duration on selectivity of neural stimulation. IEEE Trans Biomed Eng 1996;43:161-166.

15. Jacobs PL, Johnson B, Mahoney ET. Physiologic responses to electrically assisted and frame-supported standing in persons with paraplegia. J Spinal Cord Med 2003;26:384-389.

16. Jacobs PL, Mahoney ET. Peak exercise capacity of electrically induced ambulation in persons with paraplegia. Med Sci Sports Exerc 2002;34:1551-1556.

17. Jayaraman A, Gregory CM, Bowden M, Stevens J, Shah P, Behrman AL, et al. Lower extremity skeletal muscle function in persons with incomplete spinal cord injury. Spinal Cord 2006;44:680-687.

18. Mahoney ET, Bickel CS, Elder C, Black C, Slade JM, Apple D $\mathrm{Jr}$, et al. Changes in skeletal muscle size and glucose tolerance with electrically stimulated resistance training in individuals with chronic SCI. Arch Phys Med Rehabil 2005;86:1502-1504.

19. Matsunaga T, Shimada Y, Sato K. Muscle fatigue from intermittent stimulation with low and high frequency electrical pulses. Arch Phys Med Rehab 1999;80:48-53.

20. Melzack R. The McGill Pain Questionnaire: major properties and scoring methods. Pain 1975;1:277-299.

21. Russ DW, Elliot MA, Vandenborne K, Walter GA, BinderMacleod SA. Metabolic costs of isometric force generation and maintenance of human skeletal muscle. Am J Physiol Endocrinol Metab 2002;282:E448-457.

22. Scott WB, Lee SC, Johnston TE, Binkley J, Binder-Macleod SA. Contractile properties and the force-frequency relationship of the paralyzed human quadriceps femoris muscle. Phys Ther 2006;86:788-799.

23. Spinalcord: Facts and Figures at a Glance; Spinal Cord Injury Information Network, http://www.spinalcord.uab.edu/. Accessed May, 2006.

24. Thrasher A, Graham G, Popovic M. Reducing muscle fatigue due to functional electrical stimulation using random modulation of stimulation parameters. Artif Organs 2005;29:453-458.

25. Zory R, Boerio D, Jubeau M, Maffiuletti N. Central and peripheral fatigue of the knee extensor muscles induced by electromyostimulation. Int J Sports Med 2005;26:847-853. 